\title{
A Semantics-based Approach to Sensor Data Segmentation in Real-time Activity Recognition
}

\author{
Darpan Triboan ${ }^{\mathrm{a}}$, Liming Chen ${ }^{\mathrm{a}, *}$, Feng Chen $^{\mathrm{a}}$, Zumin Wang ${ }^{\mathrm{b}}$ \\ ${ }^{a}$ Context, Intelligence and Interaction Research Group, De Montfort University, UK \\ ${ }^{b}$ Department of Information Engineering, Dalian University, China
}

\begin{abstract}
Activity Recognition (AR) is key in context-aware assistive living systems. One challenge in AR is the segmentation of observed sensor events when interleaved or concurrent activities of daily living (ADLs) are performed. Several studies have proposed methods of separating and organising sensor observations and recognise generic ADLs performed in a simple or composite manner. However, little has been explored in semantically distinguishing individual sensor events directly and passing it to the relevant ongoing/new atomic activities. This paper proposes Semiotic theory inspired ontological model, capturing generic knowledge and inhabitant-specific preferences for conducting ADLs to support the segmentation process. A multithreaded decision algorithm and system prototype were developed and evaluated against 30 use case scenarios where each event was simulated at 10sec interval on a machine with i7 $2.60 \mathrm{GHz} \mathrm{CPU}, 2$ cores and 8GB RAM. The result suggests that all sensor events were adequately segmented with $100 \%$ accuracy for single ADL scenarios and minor improvement of $97.8 \%$ accuracy for composite ADL scenario. However, the performance has suffered to segment each event with the average classification time of $3971 \mathrm{~ms}$ and $62183 \mathrm{~ms}$ for single and composite ADL scenarios, respectively.
\end{abstract}

Keywords: Sensor Segmentation, User Preferences, Activities of Daily Living

\footnotetext{
${ }^{*}$ Corresponding author

Email addresses: darpan.triboan@my365.dmu.ac.uk (Darpan Triboan), liming.chen@dmu.ac.uk (Liming Chen), fengchen@dmu.ac.uk (Feng Chen), wangzumin@dlu.edu.cn (Zumin Wang)
}

Preprint submitted to Journal of ${ }^{A} T_{E} X$ Templates

July 10, 2018 
(ADL), Composite Activities, Ontology Modelling, and Activity Recognition.

\section{Introduction}

Ambient Assistive Living (AAL) systems [1, 2, 3] are being developed as a tool to support increasing ageing population [4] to carry out their Activities of Daily Living (ADL) and enable health care services to achieve higher quality5 of-care. Human Activity Recognition (HAR) is a key part of AAL systems to allow accurate and timely assistance to the inhabitant. The application of HAR approaches can also be applied to other domains such as security, surveillance, smart cities, and e-commerce. The process of activity recognition (AR) can be described in five phases described in Fig. 1

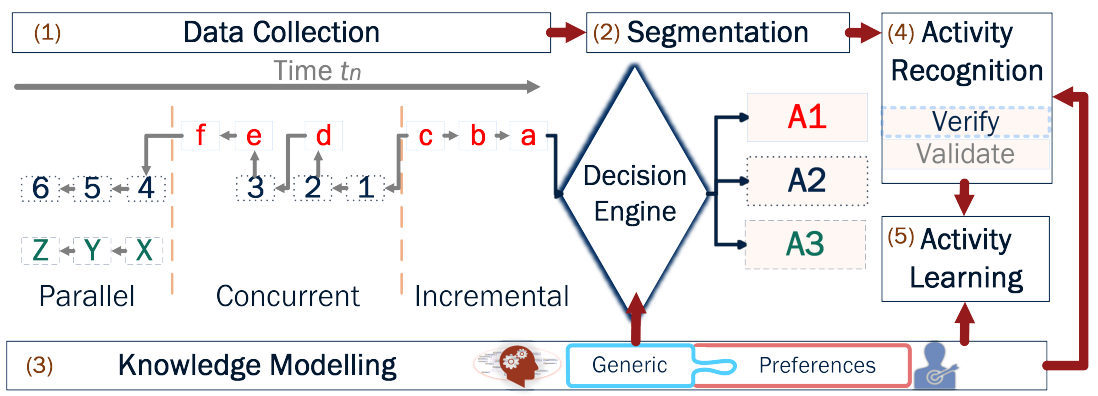

Figure 1: Five interdependent phases of AR: i) data collection, ii) segmentation of sensor observations, iii) knowledge modelling, iv) AR, and v) activity learning.

In the initial data collection phase, environmental changes and nearly every inhabitant's actions can be now sensed by the advancement of ubiquitous sensing technology. The wide variety of sensing technologies can be categorised as vision and sensor-based approaches. Whilst the vision-based sensing approach has been successfully applied in areas such as security surveillance, the sensor based approach has become more appealing in smart home ( $\mathrm{SH}$ ) environments due to lower ethical and privacy concerns. The sensor-based sensing approach can be classified into ambient, dense (or embedded) and wearable sensing [5]. The wearable sensing can be further classified into outerwear and implantable [6]. Due to such a diversity in sensors and the type of contextual data being generated 
at different frequencies simultaneously, one inherent challenge is to separate the sensor events in relation to the ongoing activity queue to later performing AR.

The second segmentation phase is responsible for organising the observed sensor events based on the ongoing activities or detecting new activities performed by a single inhabitant in composite scenarios is a major challenge being 25 investigated in this paper. In order to make segmentation decisions, prior knowledge model is required to verify association links such as what everyday object is the sensor attached to, contextual information (i.e., location, time and ambient attributes) of the object and what $\operatorname{ADL}(\mathrm{s})$ is this object is used for. The data from the set of segmented sensor observations for a given activity is later anal30 ysed by the AR algorithms to determine whether the actions were completed with a satisfactory evidence (i.e., if the cooker knob rotated to low, medium, high or off state) and provide effective assistance when necessary. Therefore, a correctly segmented set of sensors can boast AR algorithm accuracy, performance and reduces computational resources being wasted on irrelevant sensor 35 data.

The third phase is to develop a knowledge model normally based on using data-driven, knowledge-driven and hybrid approach. In the data-driven (DD) [7. 8] approach, activity models are generated after processing pre-recorded datasets using generative or discriminative classification techniques. The popu-

40 lar generative modelling methods are Bayesian networks, partial Markov decision process (POMDP) 9], a variation of Markov model to model action sequences as finite states with their transitional probabilities and continuous state-space model (CSSM) 10. Whereas, conditional random field (CRF) and support vector machine (SVM) are widely used as discriminative methods to improve the accuracy and performance of the activity recognition [11]. In contrast to DD approach, the KD approach is where domain experts in the field of interest conceptualise and intricately describe factual elements of the being into a model that is interlinked, known as the ontological model. The KD approach uses formal and logical theories to create a well-defined knowledge that 50 is based on the ontological model that is human and machine friendly to in- 
terpret. The KD approach overcomes the "cold start" issue by not processing a pre-recorded dataset, however, falls short in handling unseen or uncertain data [1. The shared problem for both of these approaches is that it assumes complete description of all the entities and concepts within the activity model.

55 Therefore, the hybrid approach [12, 13, 14] is used to combine the expressivity power from $\mathrm{KD}$ and the ability to handle unseen or uncertainty in events from the DD approach to incrementally grow the initial model.

The last two phases, activity classification and activity learning approaches [13] are influenced by the selection of modelling approach and the quality of the segmented sensor data for reasoning. Activity classification is described as a twofold process: verification of the relationships between ADLs and a set of sensor observations; and validation of the activity occurring with a degree of confidence. Whereas, the activity learning approaches evolve initial knowledge model by analysing the AR results and sensor observations to discover new activities, patterns, and inhabitant's preferences in real-time or offline. The data-driven approaches are commonly adopted for this purpose. The activity classification and activity learning topics are beyond the scope of this paper, nevertheless, for more details see [15, 16. This paper will mainly focus on verification phases of the activity classification process to reduce the computational complexity and time delay to incrementally grow the set of segmented data for a given activity as the events unfold.

There are a number of human factors that further increase the complexity when designing the semantical knowledge model, developing segmentation and AR algorithms. It is nature that one can perform single or composite (multiple) 75 ADLs at a given time as illustrated in Fig. 1. Individual ADLs (A1, A2 and A3), can have a set of atomic actions ( $\{$ abcdef $\},\{123456\}$ and $\{\mathrm{XYZ}\})$ which can be performed in any order. A single ADL (A1) can also be performed along with multiple other ADLs; either incrementally (i.e. A1 then A2), concurrently (i.e. A1 with A2), and in parallel (A2 and A3 running simultaneously). Furthermore, so an individual is subjected to follow a specific tradition, ritual or culture to perform a given activity which cannot be generalised when describing ADL. In 
addition, even when two individuals share the same values, they may still have their unique preferences to perform the same activity which can also change over time.

In the remainder of the paper, the existing studies related to segmentation, semantical knowledge modelling and AR process are reviewed in Section 2 . A novel segmentation method and algorithm is then proposed in Section 3 with system implementation details and evaluation results in Section 4 and 5 . The conclusion and future research direction is discussed in Section 6 .

\section{Related Work}

Recent studies have applied time series (fixed/dynamic time window [17, 18]), statistical and probabilistic [19] based approaches which have failed to separate sensor observations based on the relation to ongoing activity in real time. Therefore, KD approach has received an increasing amount of interest to express complex relationships between sensors and domain-specific knowledge. The process of defining complex sets of relationships has been investigated in the past studies and they can be categorised as syntactical, semantical and pragmatic in information theory [20]. In syntactical approach, a concept represented in a two or more non-syntactically equivalent statements are assumed to be statements of independent concepts. In contrary, the semantical approach is concerned about representing the meaning of a concept using relationships [20, 21, hence, the same concept can be syntactically represented in more than one statements but mean the same thing. The pragmatics approach studies the relations between a concept and inhabitant in a given context of interest. The benefit of adapting syntactical approach is that knowledge can be structured using defined syntax, queried and interpreted by the machine, however, suffer from the flexibility of expressing intricacy of relationships and meaning between two concepts that pragmatic and semantic approaches can provide. The semantic theory has its roots from semiotics in philosophy which in general is a study of signs and its 
tures and objects. Hence, the semantical theory is studied heavily in cognitive philosophy, natural language and machine learning [23]. The following sections highlight recent studies proposed to segment sensor events that adapt the notions of above three information theories.

\subsection{Semantical approaches: Indirect query and rules}

Work in [24, 25] adopted ontological models to describe ADLs, environmental entities and their relations along with other methods to classify and infer unfolding activities. However, they do not directly inspect each arrived sensor event and then segment to the appropriate queue related to ongoing activities. Instead, the continuous queries or rules are executed on events stored in the database and knowledge model without using any automatic reasoners to determine the relationship between events and ADLs. Similarly, work in [26] proposed C-SPARQL, an extension to SPARQL Protocol and RDF Query Language (SPARQL) where individual sensor events in a stream are annotated with a timestamp and continuously queried using a specific window size. The key limitations of the approach are the classical multi-query optimisation problem where the challenge is to identify the common parts, adapting/reformulating the order in which queries are executed with the ability to dynamically change the window size. Another stem of work, [27, 28, used Semantic Web Rule Language (SWRL) based inferencing rules to define the nature of activities with a temporal representation technique. These SWRL rules and Java Expert System Shell (JESS) rule engines were used to segment the sensor events using their timestamp information and perform entailments for the complexity of the ongoing activities. One of the major limitations of this approach is that an attempt to use generic ontology reasoner is made, however, it is unclear if reclassification of the whole ontology is done incrementally or not. In the case of the non-incremental reclassification approach, the performance and scalability can degrade exponentially as the size of an ontological model and data grows. Furthermore, rules can be generated for general purpose and also for inhabitant specific preferences as provided in the study in [29]. However, each time the 
new rules are added or updated to enrich the knowledge base (KB), the whole ontological model is reclassified. In addition, managing models generated using generic and inhabitant specific rules exclusively adds to the complexity further.

\subsection{Syntactical approach: $R D B M S$ and semantic KB mapping}

Similarly, work in [30] presents a layered ontology and complex event processing (CEP) engine based framework, namely, AALISABETH, to segment the sensor observations. The framework integrates temporal based reasoning with a dynamic time window sizing mechanism to segment the incoming data and perform AR in real-time. The approach leverages Esper solution for CEP and D2RQ engine to map data into RDF graphs. Although the framework utilises highly optimised, scalable Esper CEP engine solution and is open source, the system falls short in directly segmenting the incoming sensor data semantically in real-time as it arrives from the sensor network. This limits the client applications to receive an event-based notification which is critical in an emergency situation such as fall detection. Another key limitation of the framework is that the event data from the sensor network is stored directly into a traditional relational database management system (RDBMS) without inspecting individual events and segmenting them appropriately or appending to an ongoing activity queue. Instead, to filter or segment sensor events for a given ADL, continuous queries are required to be executed in order to obtain a set of sensor events between a specific time range/number of records and then perform Web Ontology Language (OWL) based reasoning capabilities to find any relevance to the activity of interest. Alternatively, the Pellet reasoner which has incremental reasoning support (i.e., only affected changes in the ontology are classified) could be further utilised instead of creating an overhead to query and map each of the events from the RDBMS database using the D2RQ tool. Furthermore, the framework is not intended to cater for inhabitant's preferences when performing a generic ADL. 


\subsection{Pragmatic approach: Precondition and evidential theory}

170 with probabilities on the phases when carrying out each ADL in order to segment the incoming events. It is unclear how the algorithm can detect new activity when an action is shared amongst more than one activities and it can either be part of a main activity or precondition actions for another activity.

Work in 31 presents an event filtering approach by adding preconditions For instance, MozzarellaCheese can be part of the precondition of MakePizza

ADL and postcondition for MakeCheesyToast ADL. This approach has achieved good accuracy in segmenting and recognising composite activities but there is the scope for improvement in terms of recognising other scenarios. Another work in 32] leveraged evidential theory and proposed three segmentation algorithms based on location, activity model and dominant-centred (key actions for a activity) for non-interleaved and interleaved activities. The location and activity model-based segmentation algorithms fall short in distinguishing activities when performed in the same location and with similar everyday objects for activities compared to the dominant algorithm. There is a little implementation 185 detail provided by the authors, however, one of the key limitations of all the three algorithms is the lack of support for user preferences and a reasoner to automatically detect and recognise the activity.

This paper makes five contributions by proposing: (i) a semantic-enabled segmentation approach which combines generic and personalised ADL knowledge that enables simple and composite ADLs to be recognised in real-time; (ii) a KB model capturing the relationships between entities in the house and ADLs; (iii) a pragmatic and light-weight mechanism to manage inhabitants specify preferences for conducting a given ADL; (iv) a semantical decision engine algorithm; (v) system implementation details and a prototype to evaluate 195 the approach and present the findings. 


\section{The Proposed Semantical Segmentation Approach}

The semantic theory based segmentation approach is proposed which analyses the relationship of the sensor event with an everyday object and its significance as an action to a set of known ADLs. This will enable disentangling composite activities with actions performed in no particular order and organise them separately to allow further activity classification and learning tasks. A knowledge modelling building block is developed in Section 3.1 which conceptualises and captures the environmental context (i.e., ambient attributes, everyday objects, location, sensors), generic and inhabitant specific preferences to perform ADLs and their semantic relationships into an ontological model. A semantical decision engine is developed in Section 3.2 to make segmentation decisions based on three inputs: the new observed sensor event, the ontological model and a set of previously segmented sensors for a given activity. A notion of multithreading is adapted to separate tasks of buffering sensor data stream, event recycling, decision engine, managing ADL threads and manipulating data from the Jena Fuseki 33. triplestore(TDB). This multithreading mechanism to semantically segment sensor event is described with a pseudo algorithm in Section 3.3 .

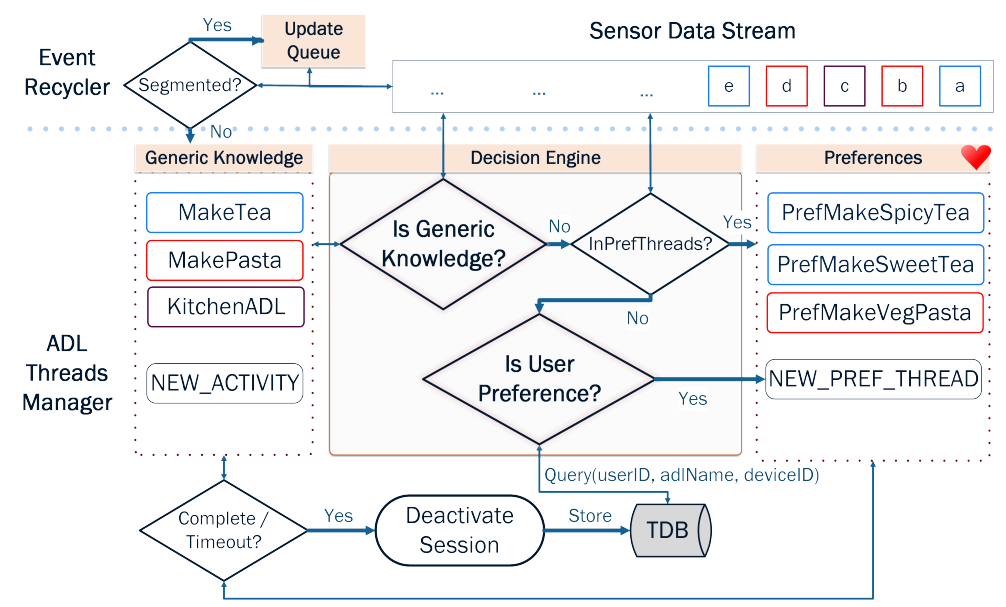

Figure 2: Overview of the semantically enabled segmentation approach with generic (T-box) and preferences (A-box) KB for reasoning. 
Fig. 2 depicts the overall segmentation approach. As the sensor events

\subsection{ADL Relationships Modelling}

The key building block of ADL modelling consists of three phases; (1) environmental context $(E C)$ modelling, $(2)$ semantical relationships $\left(S R_{i}\right)$ modelling and (3) personalised $\left(\operatorname{Pref}_{j}\right)$ object interactions. In the first phase, the object-oriented notion (classes and instances) is adapted to conceptually describe the physical or metaphysical entities $\left(E T_{k}\right)$ and their attributes as classes $(C)$ to form an overall environmental context $(E C)$ for a given smart home environment. The key entities considered are a person $\left(X_{n}\right)$, rooms (Location, 
$\left.L_{m}\right)$ and ambient characteristic $\left(A C_{p}\right)$, sensor characteristics $\left(S_{o}\right)$ and everyday fixed/portable objects $\left(O b j_{x}\right)$; see eq. 1 .

$$
E C=\left\{X_{n}, L_{m}, A C_{p}, S_{o}, O b j_{x}\right\}
$$

The second phase records semantic relationship $(S R)$ properties between $E C$ classes and ADLs. The instances of $E C$ classes (i.e., everyday objects) are then created for sensor environment $(S E)$ to create a relationship $\left(R_{e}\right)$ between sensor event, object it is attached to and this object's use in ADLs; see eq. 2 . This abstraction in ADL actions description encourages decoupling, reuse and adding the further meaning of the actions to the activity using $R_{e}$. For example, MakeTeaADL (subset of MakeHotDrinkADL) class describes the actions using hasHotDrinkType $(R)$ relationship property with Tea $(C)$ and the characteristics of the property are described to be only used for MakeHotDrinkADL (domain) and everyday objects that are used for HotDrinkType (range). This means if no other ADL that is a subset of MakeHotDrinkADL that has a hasHotDrinkType

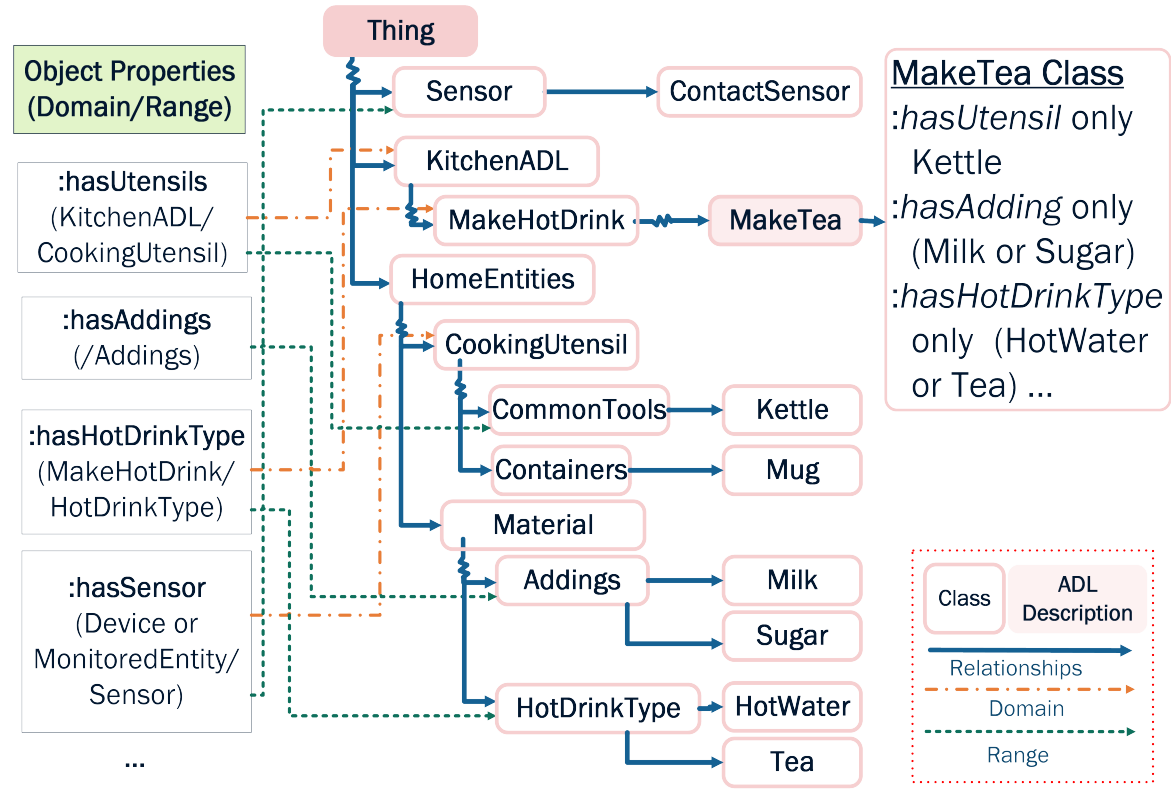

Figure 3: Semantical relationship properties between everyday objects, set of actions for MakeTea ADL and sensor characteristics. 
property with Tea, it can be deduced that this action is potentially a part of MakeTeaADL. Similarly, other actions for MakeTeaADL can be described using hasUtensil, hasContainer and hasAddings properties for using the kettle and adding sugar and milk to the teacup. Fig. 3 show the relationships between a set of EC classes and MakeTea ADL to show the meaning of inhabitant's action.

Moreover, the sensor environment $(S E)$ information is then encoded to describe existing set of $E C$ items available in the given residential environment and the sensor attached to it as instances $\left(I_{w}\right)$. Therefore, instances of $E C\left(i E C_{w}\right)$ such as environmental objects $\left(i O b j_{w}\right)$ and sensor $\left(i S_{w}\right)$ with their relevant classes $\left(C_{n}\right)$ are explicitly described with the relationship $\left(R_{e}\right)$ between them initially. For example, to 1 is an instance of ContactSensor $(S)$ that isAttachedTo $(R)$ a RedKettleObj $j_{1}\left(i O b j_{w}\right)$ which is a class type of Kettle $\left(O b j_{x}\right)$. The observed values/states of an $i S_{w}$ are stored as primitive data types $\left(p t_{u}\right)$ for a single observation or creating another instance of an observation class containing the primitive data for multiple observations; see eq. 3.

$$
\begin{gathered}
S R=A D L_{n}\left(R_{e}, E C_{n}\right) \rightarrow R_{e} \rightarrow S E \\
S E=I_{w}\left(R_{e}, S_{o}\right) \rightarrow R_{e} \rightarrow I_{w}\left(R_{e}, E T_{k}\right) \| I_{w}\left(R_{e},\left\{p t_{u}\right\}\right)
\end{gathered}
$$

The final phase is to capture inhabitant specific preferences $\left(\right.$ Pre $\left._{j}\right)$ that are subjective to individual's cultural background and rituals followed to carry out a given ADL. It is important to keep the generic (factual and commonly accepted by the wider community) and personalised sets of ADL description disjointed to avoid generalising or assuming both must be actioned to complete the activity. Therefore, instances that are members $\left(R_{e}\right)$ of Preference and $A D L_{n}$ classes are created to capture actions or ambient attributes using $i E C_{w}$ that are specific to a person $\left(X_{s}\right)$; see eq. 4 and 5 . For example, an individual $B o b(I)$ who is a type of Male $(C)$ has set of instances of Preferences that are linked with hasPreference relationship $(R)$. An example of a preference 
instance is BobMakeSpicyTeaPref (Pref) which is a type of Preference $(C)$ and MakeTeaADL $(A D L)$ with a set of $i E C$ instances, i.e., GingerObj(I) and CinnamonObj(I). This statement means that Bob has a preference to make tea and he may/like to put a ginger and cinnamon in his tea.

$$
\begin{gathered}
X_{n}=I_{w}\left(R_{e}, \text { Human } \subseteq \text { Male }\right) \rightarrow R_{e} \rightarrow \text { Pref }_{1}, \ldots \text { Pref } f_{j} \\
\text { Pref }_{j}=I_{w}\left(R_{e}, A D L_{n} \sqcap \text { Preference }\right) \rightarrow R_{e} \rightarrow I_{w}\left(R_{e}, i E C_{w}\right)
\end{gathered}
$$

\subsection{Semantic Decision Engine}

The enabling feature of the semantic-based decision engine is the ability to identify relationships between the sensor, everyday object and actions described in ADLs based on ontological model and triplestore querying. This allows decision engine to support ADL actions occurring in any order for single or multiple ADLs in a composite manner. The common ADL actions are automatically recognised using terminology box (T-box) reasoning method with incremental Pellet reasoner and inhabitant specific actions using assertion box (A-box) reasoning method. The decision engine is utilised by individual activity threads in order to find an association with new, previously observed events and candidate ADL class. The classification of candidate ADL class is continuously updated and refined with further evidence of actions that satisfies the ADL descriptions.

The decision engine takes three inputs, processes them into two stages and outputs the updated results. The three inputs are (1) semantic-based KB model created in Section 3.1. (2) activity thread $\left(A T_{n}\right)$ attempting to find relations with the (3) new sensor event $\left(e_{m}\right)$. Each $A T_{n}$ contains structured information about generic and preferred actions observed as sensor events, ADL class and list of preferences matched that are associated to the inhabitant. The twostage decision-making process updates the activity thread accordingly as the 
new sensor events are inspected incrementally for any association.

$$
\begin{aligned}
& A T_{n}=\left\{\text { tbox }\left[\text { class : some } A D L, s\left\{\ldots, e_{m}\right\}\right],\right. \\
& \quad \text { abox }\left[\text { Pref } f_{j}\left[\text { name : somePref }, s\left\{\ldots, e_{m}\right\}\right]\right\}
\end{aligned}
$$

In the first stage of the decision-making process, generic semantical relationships are traced from $E C$ to $S R$ and $S R$ to $S E$ compared to inverse when developing the KB model [34]. Therefore, the metadata of a sensor observation $e_{m}$ is analysed to find the $E T$ the sensor is attached to and deduce the potential $R_{n}$ with a set of $A D L_{n}$ description. This metadata within KB consists relationship properties such as domain and range for a given ET. Therefore, the association between $E T_{k}$, (i.e., everyday objects) and ADLs can be automatically inferred using semantic reasoners or simply querying the KB model. This process is known as terminology box (T-box) reasoning [35].

The second stage is only executed when the result returned from T-box reasoning identifies any conflicts with the ADL class description. The conflicts can be raised when a given sensor attached to an ET is forced to be part of a given ADL which is outside the restricted set of $E T_{k}$. In this case, it 320 is assumed that ET is part of inhabitant's preferences or part of a new set of actions for $A D L_{n}$. The preferences are currently pre-defined and stored as individuals containing a list of $i E C_{s}$ that an inhabitant prefers to use to perform a given $A D L$. Therefore, semantic queries are made to extract all preferences of the inhabitant (userID) for a given ADL (adlName) that as 325 sensor observation (deviceID) as an action. This process is known as assertion box (A-box) reasoning.

The semantic reasoner carries out several tasks using T-box and A-box knowledge which includes but not limited to: satisfiability, subsumption, consistency checking equivalence, disjointness, and instance checking [34, 36]. The satisfiability task is to ensure the class description (axioms) is not contradictory. The subsumption task ensures class B satisfies all the inheriting properties $(R)$ of parent class $A$. The consistency checking ensures classes and their instances 


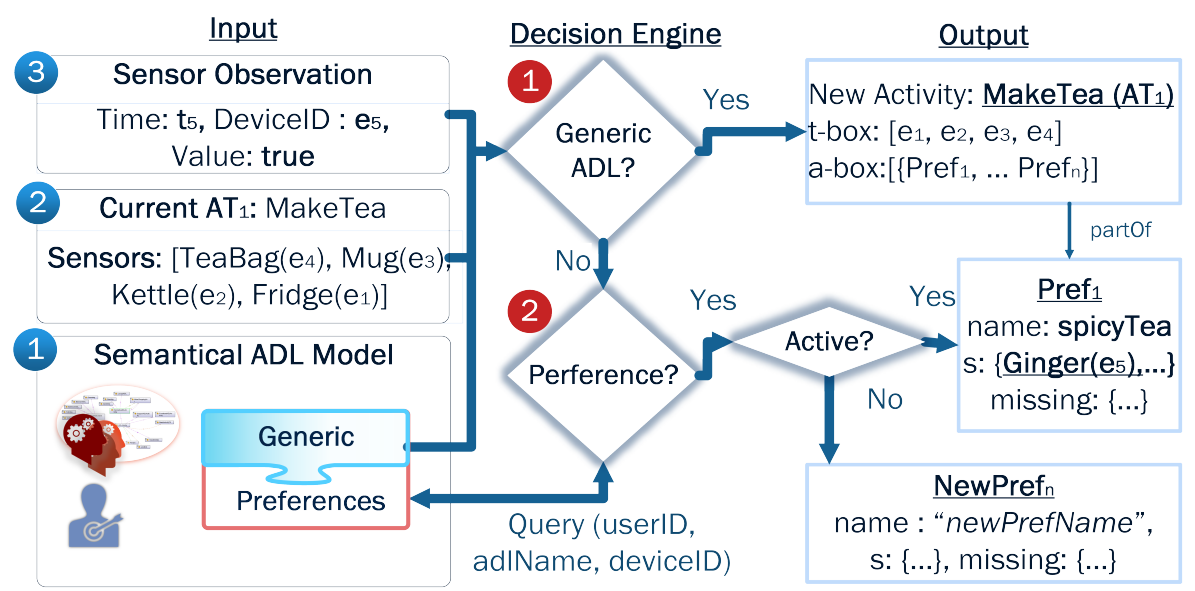

Figure 4: Semantic-based Decision Engine; Input: new sensor observation $\left(e_{5}\right)$, current activity with set of sensors and semantical ADL model, Output: new activity result

do not violate the axioms descriptions. The instance checking ensures the relationships with other instances are within the boundary of a set of classes it can subsume. The equivalence task is to match the two concepts with respect to its properties in contrary to disjointness tasks. The conjunctive querying answering is performed at the second phase of decision engine to identify inhabitant's preferences with a given $E T$ using relationships between instances of $E C$ and ADLs.

Fig. 4 illustrates the three inputs taken by the decision engine to verify if the new sensor observation Ginger $\left(e_{5}\right)$ is part of the generic/personalised action of the ongoing MakeTea activity $\left(A T_{1}\right)$. Initially, a new activity thread, $A T_{1}$, is created to add the first sensor observation, Fridge $\left(e_{1}\right)$, into the empty set of sensors and the results returned from two-stage reasoning process. In this case, ${ }_{445} e_{1}$ is inferred by the generic T-box reasoner to be part of KitchenADL in the first stage of decision engine. As the new sensor event, $e_{2}$ occurs, the current $A T_{1}$, temporarily add it to the list $\left\{e_{1}, e_{2}\right\}$ and perform the generic reasoning again with the same activity result. This means that the action is part of $A_{1}$, however, more than one sub-activities share the same actions. Similarly, other 350 events are added to $A T_{1}=\left\{e_{1}, e_{2}, e_{3}, e_{4}\right\}$ as they occurred with new MakeTea activity name which is a descendant class of MakeDrink and KitchenADL. Until 
now, only first stage of decision process is performed due to generic nature of the ADL actions. The next sensor observation, $e_{5}$, is attached to Ginger running any personalised actions. The activity name, MakeTea of $A_{1}$ and the new sensor observation Ginger $\left(e_{5}\right)$ is used to perform subsumption reasoning in the first stage of decision engine and returned inconsistency in ADL description error. In the second phase, the decision engine checks if the $\operatorname{Ginger}\left(e_{5}\right)$ sensor is part of an inhabitant's preference(s) stored in the triplestore and add it to $A_{1}$. In this case, spicyTea preference was identified and as there were no sub-activity preference threads already active for $A_{1}$, new thread Pre $f_{1}$ was created along with other missing spicyTea actions.

$$
\begin{aligned}
& A T_{1}=\left\{\text { tbox } \left\{\text { name : makeTea, } s:\left\{e_{1}, e_{2}, e_{3}, e_{4}\right\}\right.\right. \\
& \left.\quad \text { abox }\left[\text { Pref } f_{1}\left[\text { name : spicyTea, } s:\left\{e_{5}\right\}, \text { missing }:\{\ldots\}\right]\right]\right\} .
\end{aligned}
$$

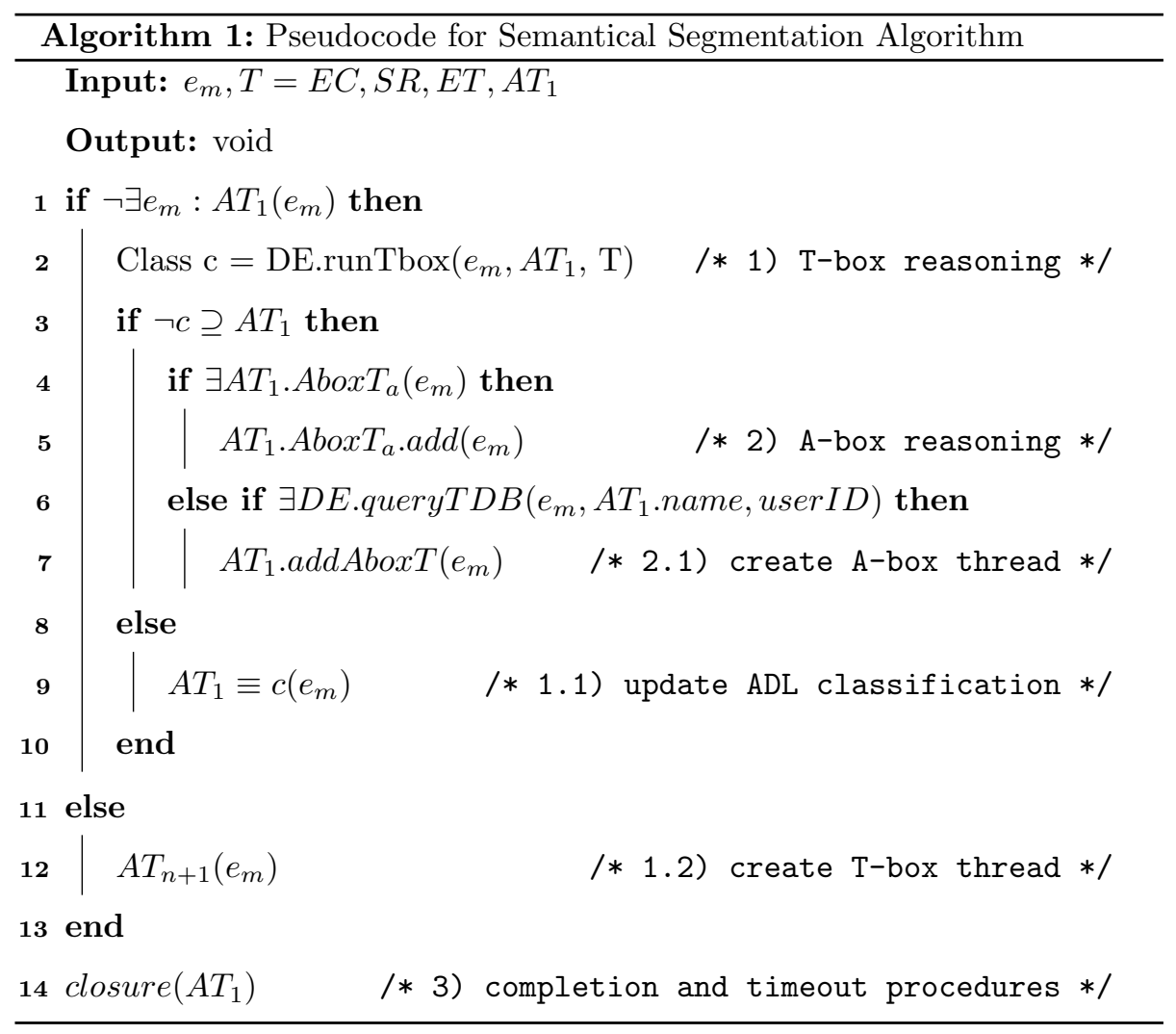




\subsection{Segmentation Algorithm}

The Algorithm 1 illustrates the segmentation process, use of decision engine

365 observations. The algorithm is performed by the ADL threads manager and it is broken down into three stages. The first stage is to iterate over all the active T-box threads $\left(A T_{n}\right)$ and use the current list of sensor observations in each thread along with the observed sensor event $\left(e_{m}\right)$ being investigated to refine a

1 shows only the first iteration $A T_{1}$ is conducted. The line 1 checks if there is $\neg \exists e_{m}$ in $A T_{1}$ then perform T-box and A-box reasoning in stage two and three. Otherwise, $e_{m}$ is assumed to be start of new ADL activity. Hence, new $A T_{n+1}$ is created with $e_{m}$ in line 12 . The T-box reasoning task in line 2 is performed by $T=\{E C, S R, E T\}$ in KB. The new deduced ADL result (Class c) is evaluated for conflicts and if $c \subseteq$ current $A T_{1}$ class then $A T_{1}$ is updated with $c$ along with $e_{m}$; see lines 3 and 9 . In the second stage, inhabitant's preferences are checked when conflicts in result is detected. All the A-box threads are checked ${ }_{380}$ if $e_{m}$ is part of active preference thread then add the event to $A b o x T_{a}$ thread. Otherwise, any inhabitant (userID) preferences $\left(A b o x T_{a}\right)$ of a given ADL class $c$ inferred for $A T_{1}$ is queried from the TDB and new A-box threads are created if matched; see lines 4-7. The final stage is where all the housekeeping for the subthreads and the process of re-evaluating the session timeout window size and timeout cases based on the data of the segmented set of observations. Details of the semantical segmentation mechanism can be found in our previous work [37, 38].

\section{System Implementation}

An android mobile application and RESTful web service have been used to 390 create a service-oriented architecture (SOA) system. An SOA enables the web service to execute computation tasks such as segmentation and AR on the sen- 


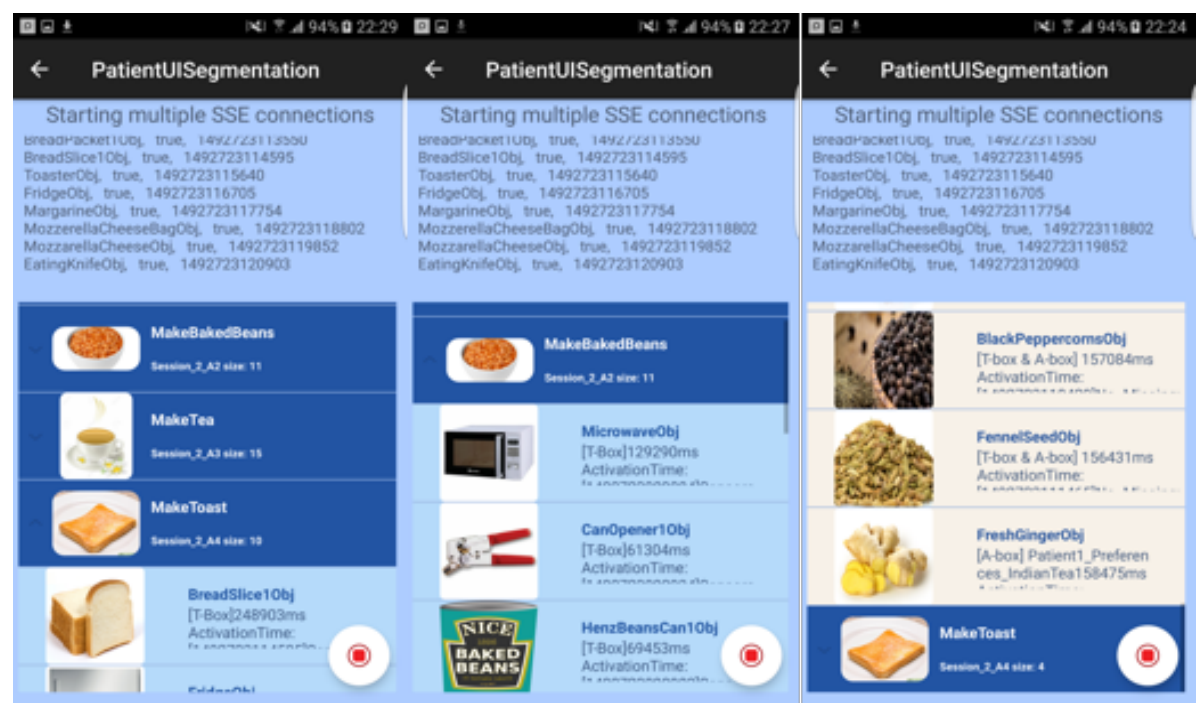

Figure 5: Segmentation results for three concurrent ADLs

sor events stream and store the results into the Jena Fuseki triplestore[33] using Jena API. The web service exposes these resources to multiple client devices running on independent operating systems using hypertext transfer protocol (HTTP) asynchronously. The web service receives all the sensor events from the sensing environment using wired/wireless connections methods and performs four main tasks; broadcast, store, segment sensor events and performs AR. The sensing environment is capable of collecting ambient data using offthe-shelf binary and multi sensors supported by Securifi Almond router with ZigBee, Z-wave and Wi-Fi communication protocol. In addition, dense sensing is supported by miniature Internet-of-Things (IoT) boards that are based on Arduino microcontroller with radio frequency (RF) and Wi-Fi capabilities to transmit and collect analogue/digital sensor data; more details in [39]. The sensor observations and the results from segmentation and AR are broadcasted independently using server-sent (SSE) protocol and stored in the Apache Jena TDB and exposed using Fuseki server 33. Multithreading concepts have been employed to segment each ADL into a thread described in Section 4.2 . A single ADL thread runs the T-Box reasoning and one or more A-Box thread(s). The reasoning result and sensor events are broadcasted to the clients and the 

partially describes the MakeTea ADL in Protégé. The MakeTea ADL class

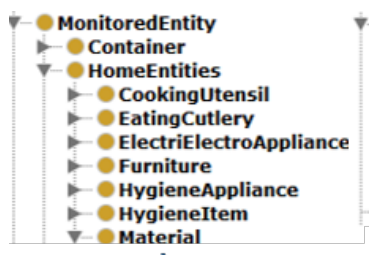

a)

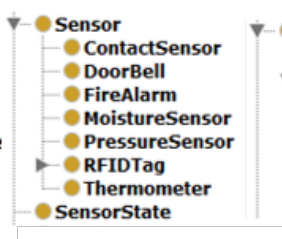

b)

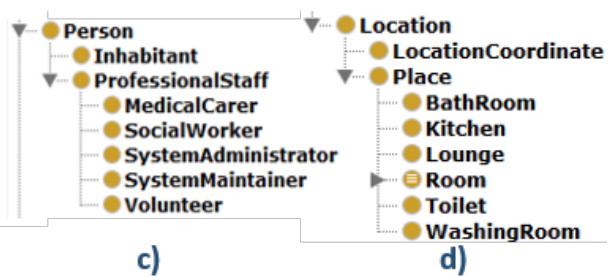

c)

d)

Figure 6: Conceptualising environmental context (EC) into Classes: a) Everyday objects $\left.\left(\operatorname{Obj}_{\mathrm{x}}\right), \mathrm{b}\right)$ Sensor characteristics $\left.\left(\mathrm{S}_{\mathrm{o}}\right), \mathrm{c}\right)$ Person $\left.\left(\mathrm{X}_{\mathrm{n}}\right), \mathrm{d}\right)$ Location $\left(\mathrm{L}_{\mathrm{m}}\right)$ 

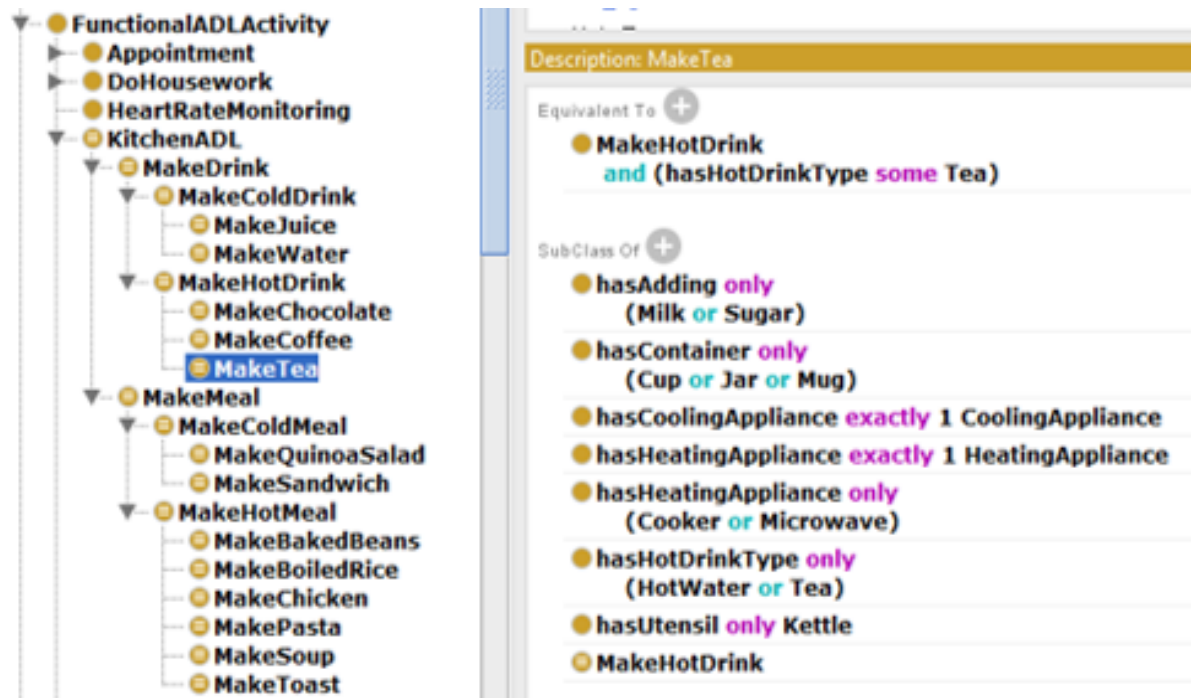

Figure 7: Partial description of MakeTea ADL with Semantic Relationship (SR) with environmental context (EC) in Protégé.

inherit the properties described from super-classes and uses $r d f s$ :subclass $O f$ object property to define actions or the context to carry out the activity. The actions properties and the classes of everyday objects for the MakeTea ADL are described using object properties hasAdding, hasContainer, hasHeatingAppliances, hasHotMealMaterial and so on. These object properties can have characteristics and relationships between everyday objects classes and the ADLs. For instance, hasHotDrinkType object property has a domain of MakeHotDrink ADL class and HotDrinkType material as range property. This means that any everyday object that is a subclass of HotDrinkType is part of the actions defined for MakeHotDrink ADL class or its subclasses. These object properties are used to add further restrictions such as universal and existential quantification $(\forall, \exists)$ using some and only, logical operations such as not, and, or $(\neg, \wedge, \vee)$, and cardinality restrictions $(\leq, \geq, \equiv)$. Other common operators are also available and can be used to increase the expressivity of the ADL model in terms of class, relationships and data. Similarly, the other 12 subclasses of MakeDrink and MakeMeal ADL classes are also described with relevant relationships. As multiple relationships with ADLs and everyday objects are created, the observed data 
(defined as individuals) with a set of assertation statements containing everyday object and object properties are used by the reasoning engine to automatically infer the type of the ADL class the actions in the individual belongs to.

Finally, the inhabitant specific preferences (A-Box) are captured by creating individuals with a direct relationship with instances of sensors in order to avoid the inconsistency in ontology description for generic knowledge. In the

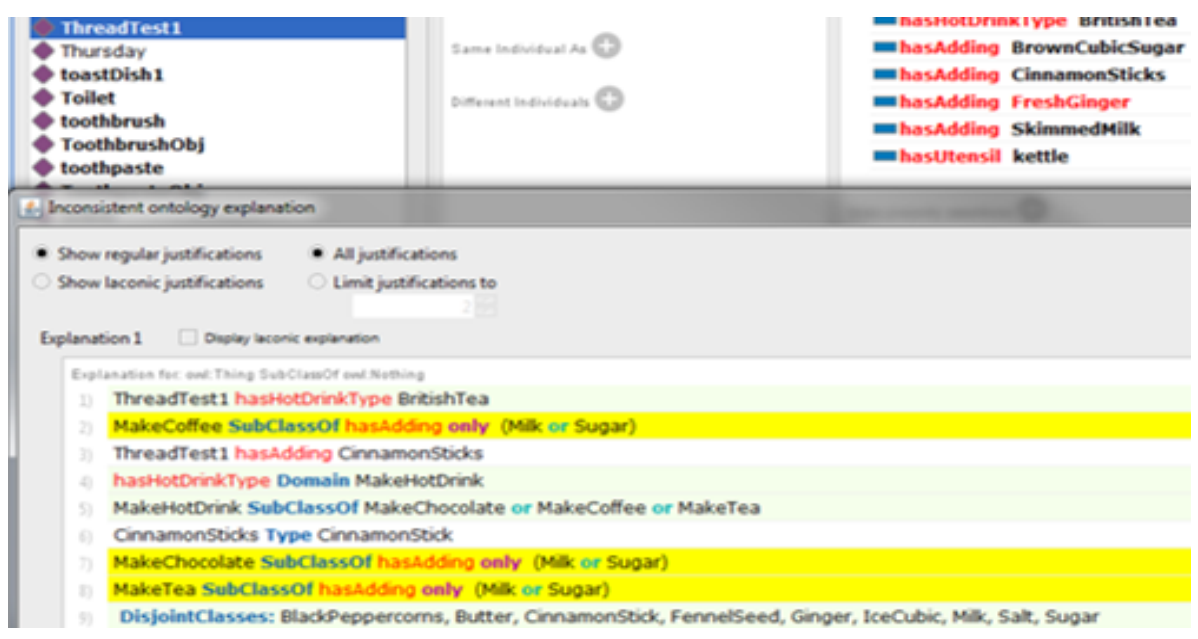

Figure 8: Inconsistency on hasAdding object property due the restriction applied to MakeTea ADL class.

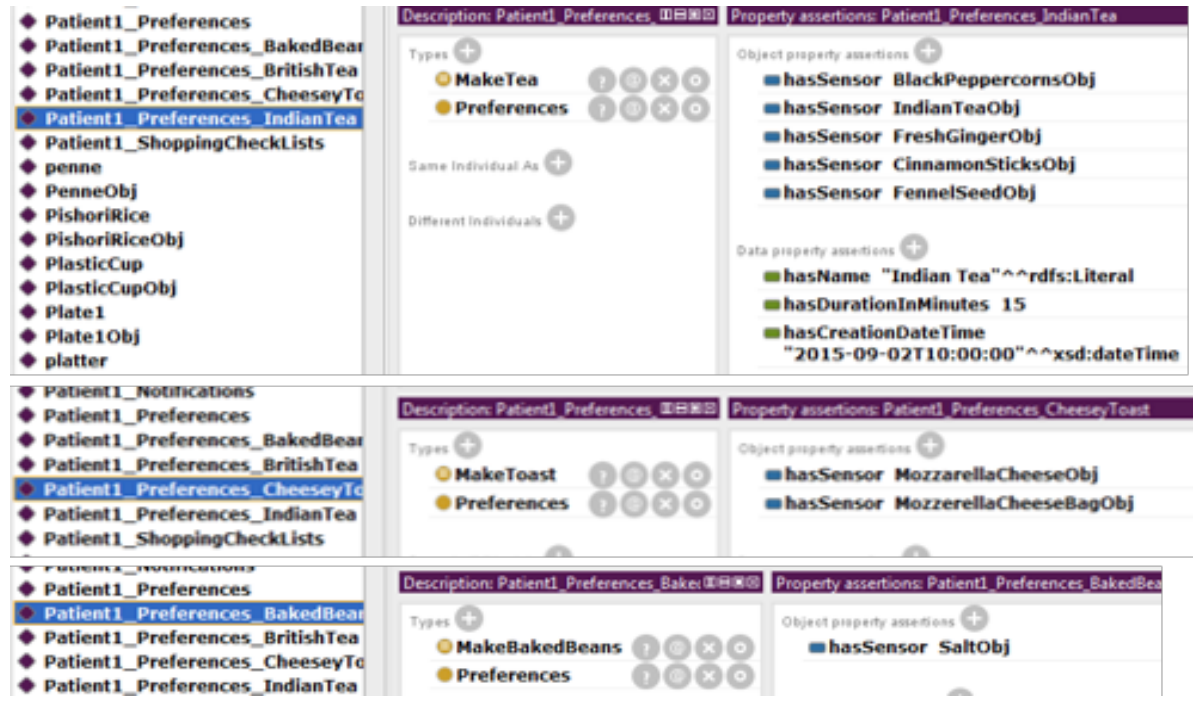

Figure 9: Inhabitant preferences as individuals with a list of sensors 
generic knowledge, not all adding (ingredient) for MakeTea ADL are defined and ingredients such as FreshGinger and CinnamonSticks are subjective to the individual. Hence, forcefully adding ingredients in an instance that is the type of MakeTea ADL will result in the inconsistent ontology as highlighted by the explanation window in Fig. 8. Therefore, instances of preferences are associated with the inhabitant and to a given ADL class which has a list of sensors that are attached to the everyday objects and other attributes. Fig. 9 presents an example of three inhabitant preferences. The top section presents individual named, Patient1_Preferences_IndianTea, which has a type of Preference class for MakeTea ADL class along with a list of sensors using hasSensor object properties and data properties to describe other attributes such as preference name and creation timestamp. Similarly, other preferences are shown in the middle and bottom of the figure to describe MakeToast and MakeBakedBeans preference.

Another method is available to layer the inhabitant specific and generic ADL ontology descriptions along with SWRL rules. This can be achieved by using the OWL API and Jena API to create and manipulate the model once generic and inhabitant specific models are combined, and rules are loaded into the memory. The reasoning can be performed using the Pellet reasoner and JESS rule engine 475 after combining the generic and inhabitant specific ontology that is managed dynamically. However, the main limitation of this method is that the changes made to the inhabitant specific ontologies will need to be tracked along with the mechanism to resolve any conflicts in the knowledge that may arise. In addition, inhabitant specific reasoner will need to be created and maintained 43 at run-time. Hence, the amount of in-memory space, number of processing cores and computation power required can grow exponentially. This can potentially create high latency in segmenting individual sensor events and undermine the scalability of the approach. Therefore, the first method is selected as it is lightweight, and no inhabitant specific reasoner is required to be running.

485 The SPARQL Inferencing Notation (SPIN) 44 rules or just a SPARQL query language can be executed on the triplestore to retrieve multiple inhabitant's 
preferences for a given ADL class simultaneously. Therefore, this method is considered appropriate during the segmentation phase as the inhabitant's preferences can be scalable and has lower latency in terms of query time and there are no additional overheads for running multiple reasoners per inhabitant.

\subsection{Multithread Segmentation Process}

The multithreaded segmentation processes are depicted in Fig. 10 where actions for MakeTea and MakeToast ADLs are performed concurrently. The generic and preferred actions are observed at a given time $\left(t_{n}\right)$. The T-box activity thread $\left(A T_{1}\right)$ is initially created when the cupObj sensor is activated at $t_{1}$. The $A T_{1}$ continuously stores the events into the thread if the decision engine infers an association with generic ADL class in the ontological model or personalised preference(s). The object attached to the cupObj sensor is queried from the triplestore, added to new individual and incremental T-box reasoning is conducted. The T-box reasoning result indicates that the object is related to ADLActivity class with no conflicts with the model, hence the A-box reasoning is not required to be executed. Next, the sensor event at $t_{2}$ is received and $A T_{1}$ performs T-box reasoning with observed sensor fridgeObj along with previous sensor(s), in this case, cupObj. The decision engine returned a new result, KitchenADL class and it was compared against the current ADLActivity class for equivalent or subsuming class. In this case, the subsuming condition is satisfied and stores the cupObj and fridgeObj sensor events in the $A T_{1}$.

Similarly, milkObj, kettleObj and indianTeaObj sensor events are processed by $A T_{1}$ where the ADL classes are incrementally classified, and the sensor events are stored in the thread. Since, the freshGingerObj sensor event is not described as part of a set of adding in the generic MakeTea ADL description, the decision engine returns with traceable conflicts. The decision engine then performs Abox reasoning to find any inhabitant's preferences related to MakeTea ADL containing freshGingerObj. Multiple preferences could be returned, in this case, only one preference named, Patient1_Pref_IndianTea $\left(P_{1}\right)$ is returned as a result of SPARQL query. A single A-box sub-thread $\left(A P T_{1}\right)$ is created with other 
missing sensors and other relevant information from the preference into the thread. The APT1 thread then inspects the incoming sensor events and updates the missing and matched sensors list independently. $A T_{1}$ thread and the subthread(s) for A-box reasoning can continue inspecting unfolding events in the data stream until the completion criteria are satisfied i.e. having no child ADL class and missing sensors in A-box threads or a dynamic timeout mechanism for the ADL. The completion/timeout criteria for the ADL will be inspected in future work.

The next set of actions for MakeToast ADL are observed between $t_{8}-t_{14}$ and inspected by $A T_{1}$ but only one shared fridgeObj event is stored. The ADL manager running in parallel inspects the sensor events in the queue and detects toastObj is not part of the MakeTea ADL class in $A T_{1}$ and $A P T_{1}$ threads. Therefore, another T-box activity thread $\left(A T_{2}\right)$ is created MakeToast ADL as depicted at the bottom-right of Fig. 10. The same process is described for AT1 is executed for the $A T_{2}$ thread to capture events from $t_{10}-t_{15}$ to $A T_{2}$ thread with one conflicting mozzarellaCheeseObj observation. Therefore, the $A P T_{2}$ thread is created when identified by decision engine that mozzarellaCheeseObj

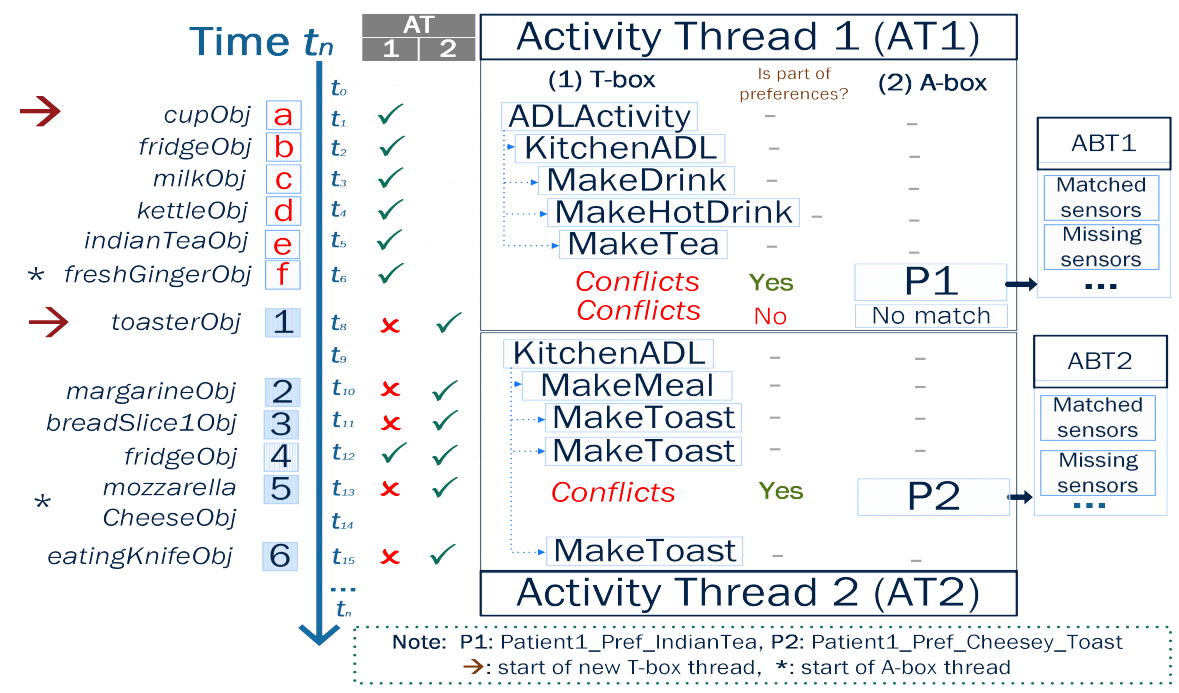

Figure 10: Concurrent actions for MakeTea and MakeToast ADL and segmentation process to create generic $\left(A T_{1}\right.$ and $\left.A T_{2}\right)$ and preference $\left(A P T_{1} \& A P T_{2}\right)$ threads when required. 
is part Patient1_Pref_CheeseyToast $\left(P_{2}\right)$ to perform the MakeToast activity. subsumption query 34 .

\section{Evaluation}

\subsection{Experiment Design}

The actions for three ADLs are scripted in no particular order to perform with only generic actions and another with the inhabitant's preferences; namely, MakeTea, MakeToast and MakeBakedBeans. The relevant actions for 
the generic $(\mathrm{G})$ ADL and some inhabitant's preferences $(\mathrm{P})$ are described in TABLE 1 These three ADLs are first tested individually in random order and then combined to create composite activity scenario; incremental, concurrent and parallel; see TABLE 2. A total of 30 activity scenarios (6 for single and 24 for composite ADLs for both $\mathrm{G}$, and $\mathrm{G}+\mathrm{P}$ actions) were created for the experiment and a thread simulated each scenario with sensor events occurring at 10ms interval. The sensor events contained a timestamp, name, sensor type, and binary data. The degree of accuracy to recognise an activity scenario is calculated in percentage by matching and tallying actual sensors events segmented correctly and it divided by the total number of sensors events activated for each ADL. The average classification time is calculated by taking sensor observation segmented time by the reasoner minus the sensor observation time recorded for each activity scenario. The unexpected sensor observations within the activity scenario are omitted and recorded separately when calculating the accuracy and average classification time for the activity. In addition, a number of duplicate activity threads created in the activity scenario are also recorded to see the effect on the overall classification times. The Samsung S6 edge smartphone running 6.0.1 Android OS was used and the web service was deployed on the HP EliteBook Folio 1040 G2 with the i7 2.60GHz processor, 2 cores, 4 logical processors and $8 \mathrm{~GB}$ RAM. The binary sensor events is currently simulated due to a limited number of sensors and time.

Table 1: Single Activity Sequences Example

\begin{tabular}{|c|c|c|c|}
\hline Activity & Type & Related actions/ sensors attached to objects & $\#$ \\
\hline Make & G & KettleObj, Cup1Obj, TeaJarObj, IndianTeaObj, KitchenSinkTap1Obj, & 9 \\
\hline Tea & $P$ & $\begin{array}{l}\text { SugarJarObj, FridgeObj, Milk1Obj, Spoon2Obj } \\
\text { [FreshGingerObj], [CinnamonSticksObj], [BlackPeppercornsObj], } \\
\text { [FennelSeedObj] }\end{array}$ & 4 \\
\hline $\begin{array}{l}\text { Make } \\
\text { Baked }\end{array}$ & G & $\begin{array}{l}\text { Spoon1Obj, HenzBeansCan1Obj, HenzBeansObj, CanOpener1Obj, } \\
\text { MicrowaveBowl1Obj, MicrowaveObj, Plate1Obj, EatingKnifeObj }\end{array}$ & 8 \\
\hline Beans & $\mathrm{P}$ & [SaltObj] & 1 \\
\hline $\begin{array}{l}\text { Make } \\
\text { Toast }\end{array}$ & G & $\begin{array}{l}\text { Plate1Obj, BreadPacket1Obj, BreadSlice1Obj, ToasterObj, FridgeObj, } \\
\text { MargarineObj, EatingKnifeObj }\end{array}$ & 7 \\
\hline & $\mathrm{P}$ & [MozzerellaCheeseBagObj], [MozzarellaCheeseObj] & 2 \\
\hline
\end{tabular}


Table 2: Combinations of Simple activities

\begin{tabular}{|c|c|c|c|c|}
\hline \multirow{2}{*}{$\begin{array}{l}\text { Activity } \\
\text { Comb. }\end{array}$} & \multirow[t]{2}{*}{ ADL Sequences } & \multirow{2}{*}{$\begin{array}{l}\text { Expected } \\
\text { no. } \\
\text { threads }\end{array}$} & \multicolumn{2}{|c|}{ Actions } \\
\hline & & & Gen. (G) & $\begin{array}{l}+ \text { pref. } \\
(\mathrm{G}+\mathrm{P})\end{array}$ \\
\hline AC1 & MakeTea, MakeToast & 2 & 16 & 22 \\
\hline $\mathrm{AC} 2$ & $\begin{array}{l}\text { MakeTea, } \\
\text { MakeBakedBeans }\end{array}$ & 2 & 17 & 22 \\
\hline $\mathrm{AC} 3$ & $\begin{array}{l}\text { MakeToast, } \\
\text { MakeBakedBeans }\end{array}$ & 2 & 15 & 18 \\
\hline $\mathrm{AC4}$ & $\begin{array}{l}\text { MakeToast, } \\
\text { MakeBakedBeans, } \\
\text { MakeTea }\end{array}$ & 3 & 24 & 31 \\
\hline AC5 & $\begin{array}{l}\text { MakeBakedBeans, } \\
\text { MakeTea, MakeToast }\end{array}$ & 3 & 24 & 31 \\
\hline AC6 & $\begin{array}{l}\text { MakeTea, MakeToast, } \\
\text { MakeBakedBeans }\end{array}$ & & 24 & 31 \\
\hline Total & & 15 & 120 & 155 \\
\hline
\end{tabular}

\subsection{Results}

The average segmentation time taken per sensor event for single activity

is $3971 \mathrm{~ms}$ in contrast to $62183 \mathrm{~ms}$ for composite ADL scenarios as shown in TABLE 3 and TABLE 4 The result in TABLE 3 shows that all the sensor events for a single activity case scenario were adequately placed in the correct thread with $100 \%$ accuracy. Only the MakeTea activity case scenario created additional threads with more than double the average time when processing 9 generic actions and 4 preferred actions. On the other hand, TABLE 4 shows 20 out of 24 activities performed in a composite manner or 572 out of 585 sensor events were added to the relevant thread, giving $97.8 \%$ accuracy. However,

Table 3: Single Activity performed in no Specific order with Generic and Personal Preferences

\begin{tabular}{|c|c|c|c|c|c|}
\hline Activity & Type & $\begin{array}{l}\text { In relevant } \\
\text { thread }\end{array}$ & $\begin{array}{l}\text { Unexp. actions } \\
\text { in thread(s)* }\end{array}$ & $\begin{array}{l}\text { Excess } \\
\text { thread (s) }\end{array}$ & $\begin{array}{l}\text { Avg. time } \\
\text { (ms) + }\end{array}$ \\
\hline MakeTea & G & 9 & 0 & 0 & 2394.67 \\
\hline MakeToast & G & 7 & 0 & 0 & 2468.57 \\
\hline $\begin{array}{l}\text { MakeBaked } \\
\text { Beans }\end{array}$ & & 8 & 0 & 0 & 2372.25 \\
\hline MakeTea & $\mathrm{G}+\mathrm{P}$ & 13 & 0 & 1 & 10828.85 \\
\hline MakeToast & $\mathrm{G}+\mathrm{P}$ & 9 & 0 & 0 & 3786.87 \\
\hline $\begin{array}{l}\text { MakeBaked } \\
\text { Beans }\end{array}$ & $\mathrm{G}+\mathrm{P}$ & 9 & 0 & 0 & 1972.44 \\
\hline $\begin{array}{l}\text { Total } \\
\text { Note: } \\
\text { threads }\end{array}$ & $\begin{array}{l}6 \\
\text { cludes }\end{array}$ & $\begin{array}{l}55 / 55 \\
\text { additional }\end{array}$ & $\begin{array}{l}0 \\
\text { thread(s) acti }\end{array}$ & $\begin{array}{l}1 \\
\text { ons, }+ \text { in }\end{array}$ & $\begin{array}{l}3970.61 \text { (avg) } \\
\text { cluding excess }\end{array}$ \\
\hline
\end{tabular}


Table 4: Multiple activities performed in a composite manner

\begin{tabular}{|c|c|c|c|c|c|c|}
\hline & $\begin{array}{l}\text { Activity } \\
\text { Comb. }\end{array}$ & Type & $\begin{array}{l}\text { All actions in } \\
\text { the thread(s)? }\end{array}$ & $\begin{array}{l}\text { Excess } \\
\text { thread(s) }\end{array}$ & $\begin{array}{l}\text { Unexp. actions } \\
\text { in the thread(s)* }\end{array}$ & $\begin{array}{l}\text { Total Avg. } \\
\text { time+ (ms) }\end{array}$ \\
\hline \multirow[t]{12}{*}{ Inc. } & $\mathrm{ACl}$ & G & $\checkmark \quad 16$ & 1 & 1 & 36330.64 \\
\hline & $\mathrm{AC2}$ & G & 17 & 1 & 4 & 41543.17 \\
\hline & $\mathrm{AC} 3$ & G & 15 & 1 & 1 & 30354.98 \\
\hline & $\mathrm{AC} 4$ & G & $15 / 24$ & 3 & 3 & 95819.25 \\
\hline & $\mathrm{AC5}$ & G & 24 & 1 & 5 & 60742.14 \\
\hline & AC6 & G & $\checkmark 24$ & 1 & 6 & 72690.97 \\
\hline & $\mathrm{AC} 1$ & $\mathrm{G}+\mathrm{P}$ & $\checkmark \quad 22$ & 1 & 1 & 54949.21 \\
\hline & $\mathrm{AC2}$ & $G+P$ & $\checkmark \quad 22$ & 0 & 5 & 21905.05 \\
\hline & $\mathrm{AC} 3$ & $G+P$ & $\checkmark \quad 18$ & 0 & 1 & 12561.28 \\
\hline & $\mathrm{AC} 4$ & $G+P$ & $\times \quad 31$ & 3 & 3 & 99807.19 \\
\hline & $\mathrm{AC5}$ & $G+P$ & $\times \quad 30 / 31$ & 1 & 4 & 62016.20 \\
\hline & AC6 & $\mathrm{G}+\mathrm{P}$ & $\checkmark \quad 31$ & 1 & 3 & 87298.32 \\
\hline \multirow{6}{*}{ Con. } & $\mathrm{AC1}$ & $G+P$ & $\checkmark \quad 22$ & 1 & 0 & 56752.83 \\
\hline & $\mathrm{AC2}$ & $G+P$ & $\checkmark 22$ & 1 & 5 & 23993.51 \\
\hline & $\mathrm{AC} 3$ & $G+P$ & $\checkmark \quad 18$ & 2 & 1 & 64074.61 \\
\hline & $\mathrm{AC} 4$ & $G+P$ & $\checkmark \quad 31$ & 1 & 1 & 70289.79 \\
\hline & $\mathrm{AC5}$ & $G+P$ & $\checkmark \quad 31$ & 2 & 6 & 131784.92 \\
\hline & AC6 & $G+P$ & 31 & 2 & 5 & 181894.97 \\
\hline \multirow{7}{*}{ Par. } & $\mathrm{AC} 1$ & $G+P$ & $21 / 22$ & 2 & 0 & 43055.55 \\
\hline & $\mathrm{AC2}$ & $G+P$ & $\checkmark 22$ & 0 & 3 & 8309.10 \\
\hline & $\mathrm{AC} 3$ & $G+P$ & $16 / 18$ & 1 & 0 & 35944.94 \\
\hline & $\mathrm{AC} 4$ & $G+P$ & $\checkmark \quad 31$ & 1 & 4 & 63737.04 \\
\hline & AC5 & $G+P$ & $\checkmark \quad 31$ & 1 & 5 & 77355.87 \\
\hline & AC6 & $G+P$ & $\checkmark \quad 31$ & 1 & 4 & 59173.90 \\
\hline & Total & 24 & $572 / 585$ & 29 & 71 & 62182.73 (avg \\
\hline
\end{tabular}

the segmented activity threads captured a total of 71 additional unexpected sensor events in the segmented threads which are not necessarily incorrect, i.e., multiple spoon objects or heating/cooling appliances when performing multiple activities interweavingly. Furthermore, 29 additional threads were created and failed to classify any ongoing activity.

\subsection{Discussion}

Althought, previous studies use varying ADL models, datasets, sensors and platforms, use scenarios, and etc., the key features and final outcomes for the recent KD studies presented in Section 2 is discussed instead. The accuracy of single and composite activity segmentation for evidential theory-based approach [32 is $81.8 \%$ and $76.2 \%$ on average and ontology and temporal 28, achieved $100 \%$ and $88.3 \%$, respectively. Therefore, there is a significant evidence that 605 the proposed approach improves the accuracy of sensor segmentation with $100 \%$ 
and $97.8 \%$, respectively. In addition, user-preferences are taken into consideration by adopting basic query based approach and automatic Pellet reasoner for generic KB reasoning compared to their counterparts which adapt solely querybased approach inheriting classical multi-query optimisation problem in [26] and [30]. Nevertheless, one of the benefits for adapting multi-query approach is that higher performance and scalability can be achieved, however, suffer from the expressivity capabilities of KB due to explicit query development/maintenance efforts and the ability to use automatic reasoners.

The proposed method in this paper seeks to strike a balance between automation by taking advantage of expesive ontology with incremental Pellet reasoning feature and performance of query-based approach to manage the changing userpreferences. The average segmentation time information is not available in the presented KB studies; however, the proposed approaches observes 3971ms and $62183 \mathrm{~ms}$ with sensors events activated at the 10s interval for simple and composite activities scenarios. These results are still not suitable for the real-time system at this stage. However, the optimisation opportunities such as multithread safe reasoning [46], ADL threads management, parallel programming, partitioning workload to graphics processing units (GPUs) [4], and using a

Table 5: Summary of recent KB approaches

\begin{tabular}{|c|c|c|c|c|c|}
\hline $\begin{array}{l}\text { Studies } \\
\text { /Features }\end{array}$ & $\begin{array}{l}\text { C-SPARQL } \\
{[24], 2010}\end{array}$ & $\begin{array}{l}\text { Evidential theory } \\
\text { [30], } 2013\end{array}$ & $\begin{array}{l}\text { Onto. \& temporal } \\
\text { [26], } 2014\end{array}$ & $\begin{array}{l}\text { AALISABETH } \\
{[28], 2015}\end{array}$ & Proposed \\
\hline $\begin{array}{l}\text { Knowledge } \\
\text { expressivity }\end{array}$ & High & High & High & High & High \\
\hline $\begin{array}{l}\text { SPARQL query } \\
\text { support }\end{array}$ & Yes & Yes & Yes & Yes & Yes \\
\hline $\begin{array}{l}\text { Automatic } \\
\text { reasoner support }\end{array}$ & No & No & Yes & No & Yes \\
\hline $\begin{array}{l}\text { Direct stream } \\
\text { inspection }\end{array}$ & No & Yes & Yes & No & Yes \\
\hline RDF stored & Yes & NA & Yes & No & Yes \\
\hline $\begin{array}{l}\text { User prefs. } \\
\text { support }\end{array}$ & No & No & No & No & Yes \\
\hline $\begin{array}{l}\text { Sliding window } \\
\text { support }\end{array}$ & $\begin{array}{l}\text { Yes } \\
\text { (Fixed size) }\end{array}$ & No & Yes & Yes & $\begin{array}{l}\text { No } \\
\text { (Future work) }\end{array}$ \\
\hline $\begin{array}{l}\text { Potential } \\
\text { scalability issue }\end{array}$ & Low & Med. - High & Med. & Low & Med. - High \\
\hline Accuracy: S; C (\%) & - & $81.8 ; 76.2$ & $100 ; 88.3$ & - & $100 ; 97.8$ \\
\hline $\begin{array}{l}\text { Average time: } \\
\mathrm{S} ; \mathrm{C}(\mathrm{ms})\end{array}$ & - & - & - & - & $3971 ; 62183$ \\
\hline
\end{tabular}


machine with higher number of cores (i.e., quad-core, octa-core CPU or higher)

to support more concurrent or parallel threads execution at same time remain an open challenge. TABLE 5 presents a summary of the key components of the recent KB studies presented in Section 2 against the proposed semantical segmentation approach in this paper.

\section{Conclusion and Future Work}

A semantical segmentation approach is proposed which combines generic knowledge conceptualised as an ontological model and inhabitant specific preferences to conduct a specific ADL as asserted individual. Upon sensor activation, the event is inspected by one or more active $\mathrm{ADL}$ threads running in parallel. Each ADL thread relies on a two-stage decision engine to find any association with observed sensor event. The decision engine conducts T-box reasoning with generic $\mathrm{KB}$ in the first stage and A-box reasoning with observed sensor event and inhabitant specific preferences by querying the triplestore in the second stage. The second stage of decision engine is only invoked when the use of entity on which observed sensor is attached to has a contradiction or not been explicitly specified in generic ADL description. The ADL thread discards the observed event when decision engine has failed to find any relationship. When the whole set of active ADL threads fail to find any relevance for a given sensor event, a new ADL thread is created. The approach leverages between the incremental Pellet reasoner, OWL \& Jena API, and the notion of multithreading. The proposed method was implemented and tested against 30 test scenarios. The results indicate an improvement in segmentation accuracy compared to the counterpart studies with $100 \%$ and $88.3 \%$ for single and composite ADL scenarios with an average time of $3971 \mathrm{~ms}$ and $62183 \mathrm{~ms}$. The main bottlenecks for high processing time are the synchronised incremental reasoning and duplicate ADL threads creation which ultimately created additional reasoning tasks and slowed down the overall process on the machine which was limited to two cores. A future study is proposed to address above shortfalls by adapting 
Fork/Join parallelism framework [48] to efficiently split and manage tasks over multiple cores machine and utilise graphical processing unit (GPU) to increase performance. Moreover, investigating in methods for making incremental Pellet reasoner thread-safe and allow parallel processing can encourage more real-time scalable solutions to emerge. Finally, the study will focus on comparing other segmentation approaches, developing accurate fine-grained AR and learning algorithms with the support of the rule and temporal reasoning.

\section{References}

[1] S. Zolfaghari, R. Zall, M. R. Keyvanpour, SOnAr: Smart Ontology Activity recognition framework to fulfill Semantic Web in smart homes Samaneh, in: Proceedings of the Annual Hawaii International Conference on System Sciences, Vol. 2016-March, 2016, pp. 3339-3348. doi:10.1109/HICSS.2016. 417.

[2] C. Debes, A. Merentitis, S. Sukhanov, M. Niessen, N. Frangiadakis, A. Bauer, Monitoring Activities of Daily Living in Smart Homes, IEEE Signal Processing Magazine (March) (2016) 81-94. doi:10.1109/MSP. 2015. 2503881 .

[3] Z. Tang, J. Guo, S. Miao, S. Acharya, J. H. Feng, Ambient intelligence based context-aware assistive system to improve independence for people with autism spectrum disorder, Proceedings of the Annual Hawaii International Conference on System Sciences 2016-March (2016) 3339-3348. doi:10.1109/HICSS.2016.417.

[4] B. J. Banks, The "Age" of Opportunity, IEEE Pulse 8 (2) (2017) 12-15.

[5] C. Ye, Y. Xia, Y. Sun, S. Wang, H. Yan, R. Mehmood, ERAR: An EventDriven Approach for Real-Time Activity Recognition, 2015 International Conference on Identification, Information, and Knowledge in the Internet of Things (IIKI) (2015) 288-293doi:10.1109/IIKI.2015.69. 
[10] Q. Ding, J. Han, X. Zhao, Continuous estimation of human multi-joint angles from semg using a state-space model, IEEE Transactions on Neural

1. Systems and Rehabilitation Engineering 25 (9) (2017) 1518-1528. doi: 10.1109/TNSRE. 2016.2639527.

[11] T. Nef, P. Urwyler, M. Büchler, I. Tarnanas, R. Stucki, D. Cazzoli,

URL http://ieeexplore.ieee.org/lpdocs/epic03/wrapper.htm? arnumber $=7428373$

[6] M. Cornacchia, Y. Zheng, A Survey on Activity Detection and Classification Using Wearable Sensors, IEEE SENSORS JOURNAL 17 (2) (2017) 386-403.

[7] L. Chen, C. D. Nugent, H. Wang, A knowledge-driven approach to activity recognition in smart homes, IEEE Transactions on Knowledge and Data Engineering 24 (6) (2012) 961-974. doi:10.1109/TKDE.2011.51.

[8] L. Chen, C. Nugent, G. Okeyo, An ontology-based hybrid approach to activity modeling for smart homes, IEEE Transactions on Human-Machine Systems 44 (1) (2014) 92-105. doi:10.1109/THMS .2013.2293714

[9] E. M. D. Jean-Baptiste, A. Mihailidis, Analysis and comparison of two task models in a partially observable markov decision process based assistive system, in: 2017 IEEE 4th International Conference on Soft Computing Machine Intelligence (ISCMI), 2017, pp. 183-187. doi:10.1109/ISCMI. 2017.8279623

R. Müri, U. Mosimann, Evaluation of Three State-of-the-Art Classifiers for Recognition of Activities of Daily Living from Smart Home Ambient Data., Sensors (Basel, Switzerland) 15 (5) (2015) 11725-40. doi:10.3390/s150511725. URL http://www.scopus.com/inward/record.url?eid=2-s2. 0-84930640821\{\&\}partnerID=tZ0tx3y1 
[12] L. Chen, C. Nugent, J. Rafferty, Ontology-based Activity Recognition Framework and Services, Proceedings of International Conference on Information Integration and Web-based Applications \& Services - IIWAS '13 (2013) 463-469doi:10.1145/2539150.2539187. URL http://doi.acm.org/10.1145/2539150.2539187\{\%\}5Cnhttp: //dl.acm.org/citation. $\mathrm{cfm}$ ?doid=2539150.2539187

[13] L. Chen, G. Okeyo, H. Wang, R. Sterritt, C. Nugent, A systematic approach to adaptive activity modeling and discovery in smart homes, Proceedings - 2011 4th International Conference on Biomedical Engineering

口 and Informatics, BMEI 20114 (2011) 2192-2196. doi:10.1109/BMEI. 2011.6098760

[14] P. Barnaghi, W. Wang, L. Dong, C. Wang, A linked-data model for semantic sensor streams, Proceedings - 2013 IEEE International Conference on Green Computing and Communications and IEEE Internet of Things and IEEE Cyber, Physical and Social Computing, GreenCom-iThings-CPSCom 2013 (2013) 468-475doi:10.1109/GreenCom-iThings-CPSCom.2013.95

[15] D. Cook, K. Feuz, N. Krishnan, Transfer Learning for Activity Recognition: A Survey, Knowledge and Information Systems 36 (3) (2013) 537-556. doi:10.1007/s10115-013-0665-3. URL http://eecs .wsu.edu/\{ $\} \operatorname{cook} /$ pubs/kais12.pdf

[16] O. Brdiczka, J. L. Crowley, P. Reignier, Learning situation models in a smart home, IEEE Transactions on Systems, Man, and Cybernetics, Part B: Cybernetics 39 (1) (2009) 56-63. doi:10.1109/TSMCB.2008.923526

[17] G. Okeyo, L. Chen, H. Wang, R. Sterritt, Dynamic sensor data segmentation for real time activity recognition, Pervasive and Mobile Computing 10, Part B (2014) 155-172.

11 URL http://www.tech.dmu.ac.uk/\{ \}limingchen/PMC-S-11-00304.

a pdfhttp://www.sciencedirect.com/science/article/pii/ $735 \quad$ S1574119212001393 
[18] J. Wan, M. J. O’Grady, G. M. P. O'Hare, Dynamic sensor event segmentation for real-time activity recognition in a smart home context, Personal and Ubiquitous Computing 19 (2) (2015) 287-301. doi:10.1007/ s00779-014-0824-x. 口1

[19] D. R. Faria, M. Vieira, C. Premebida, U. Nunes, Probabilistic Human Daily Activity Recognition towards Robot-assisted Living (2015) 582-587.

[20] Y. Zhong, A theory of semantic information, China Communications 14 (1) (2017) 1-17. doi:10.1109/CC.2017.7839754

[21] A. Tarski, The Semantic Conception of Truth: and the Foundations of Semantics (1944). doi:10.2307/2102968. URL http://www.jstor.org/stable/2102968?origin=crossref

[22] P. Vickers, Understanding Visualisation: A Formal Foundation using Category Theory and Semiotics, IEEE Transactions on Visualization and Computer Graphics X (X) (2013) 1-14.

[23] Y. Wang, Formal rules for concept and semantics manipulations in cognitive linguistics and machine learning, in: 2017 IEEE 16th International Conference on Cognitive Informatics Cognitive Computing (ICCI*CC), 2017, pp. 43-50. doi:10.1109/ICCI-CC.2017.8109728.

[24] J. Rafferty, C. D. Nugent, J. Liu, L. Chen, From Activity Recognition to Intention Recognition for Assisted Living Within Smart Homes, IEEE Transactions on Human-Machine Systems PP (99) (2016) 1-12. doi:10. 1109/THMS.2016.2641388.

URL http://ieeexplore.ieee.org/document/7807210/

[25] G. Meditskos, S. Dasiopoulou, I. Kompatsiaris, MetaQ: A knowledgedriven framework for context-aware activity recognition combining SPARQL and OWL 2 activity patterns, Pervasive and Mobile Computingdoi:10.1016/j.pmcj.2015.01.007.

URL http://wWw.sciencedirect.com/science/article/pii/ 


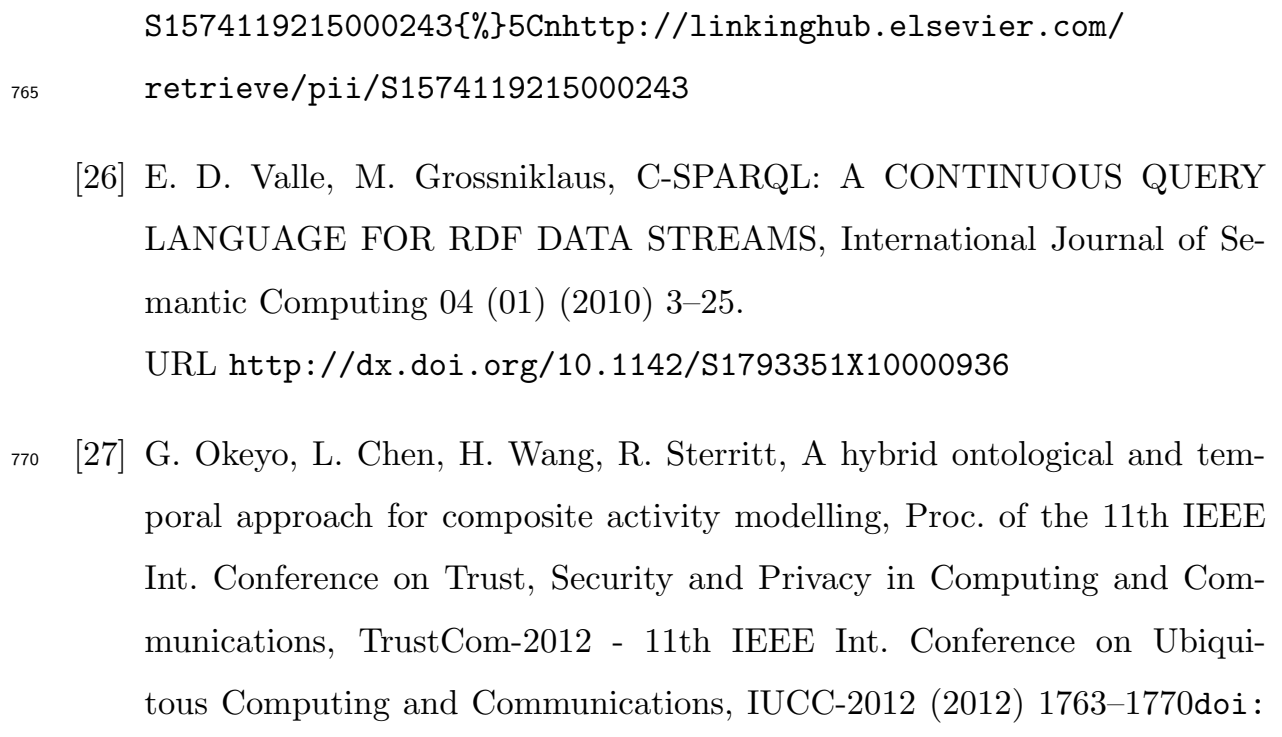

[26] E. D. Valle, M. Grossniklaus, C-SPARQL: A CONTINUOUS QUERY LANGUAGE FOR RDF DATA STREAMS, International Journal of Semantic Computing 04 (01) (2010) 3-25.

URL http://dx.doi.org/10.1142/S1793351X10000936

[30] R. Culmone, P. Giuliodori, M. Quadrini, Human Activity Recognition using a Semantic Ontology-Based Framework, International Journal on Advances in Intelligent Systems 8 (2) (2015) 159-168.

[

790

[31] U. Naeem, Activities of daily life recognition using process representation 
modelling to support intention analysis, International Journal of Pervasive Computing and Communications 11 (3) (2015) 347. doi:10.1108/ IJPCC-01-2015-0002.

[38] D. Triboan, L. Chen, F. Chen, S. Fallmann, I. Psychoula, Real-Time Sensor Observation Segmentation For Complex Activity Recognition Within Smart Environments, in: 2017 IEEE 14th International Conference on Ubiquitous Intelligence and Computing (UIC 2017), San Francisco, 2017. 
[39] D. Triboan, L. Chen, F. Chen, Z. Wang, Towards a Service-Oriented Architecture for a Mobile Assistive System with Real-time Environmental Sensing, TSINGHUA SCIENCE AND TECHNOLOGY 21 (6) (2016) 581597.

[40] D. Triboan, L. Chen, F. Chen, Towards a Mobile Assistive System Us825 ing Service- oriented Architecture, in: 2016 IEEE Symposium on ServiceOriented System Engineering Towards, IEEE, Oxford, 2016, pp. 187-196. doi:10.1109/SOSE. 2016.41.

URL http://doi .ieeecomputersociety.org/10.1109/SOSE.2016.41

[41] D. Riboni, C. Bettini, OWL 2 modeling and reasoning with complex human 830 activities, Pervasive and Mobile Computing 7 (3) (2011) 379-395. doi: $10.1016 / j \cdot p m c j \cdot 2011.02 .001$. URL http://dx.doi.org/10.1016/j.pmcj.2011.02.001

[42] S. University, Protégé.

URL https://protege.stanford.edu/

[43] R. Volz, S. Staab, B. Motik, Incremental Maintenance Of Materialized Ontologies, Lecture Notes in Computer Science 2888/2003 (2888/2003) (2003) $707-724$.

[44] W3C, SPIN - Overview and Motivation (2011). URL https://www.w3.org/Submission/spin-overview/

[45] B. Cuenca Grau, C. Halaschek-Wiener, Y. Kazakov, History matters: Incremental ontology reasoning using modules, Lecture Notes in Computer Science (including subseries Lecture Notes in Artificial Intelligence [ and Lecture Notes in Bioinformatics) 4825 LNCS (2007) 183-196. doi: 10.1007/978-3-540-76298-0_14.

${ }_{845}^{646]}$ Y. Ren, J. Z. Pan, I. Guclu, M. Kollingbaum, A combined approach to incremental reasoning for EL ontologies, Lecture Notes in Computer Science (including subseries Lecture Notes in Artificial Intelligence and 
Lecture Notes in Bioinformatics) 9898 LNCS (August) (2016) 167-183. doi:10.1007/978-3-319-45276-0_13.

[47] M. Peters, C. Brink, S. Sachweh, A. Zündorf, Scaling parallel rule-based reasoning, Lecture Notes in Computer Science (including subseries Lecture Notes in Artificial Intelligence and Lecture Notes in Bioinformatics) 8465 LNCS (2014) 270-285. doi:10.1007/978-3-319-07443-6_19.

[48] J. Ponge, Fork and Join: Java Can Excel at Painless Parallel Programming 855 Too! (2011).

【 $\mathrm{URL}$ http://www.oracle.com/technetwork/articles/java/
fork-join-422606.html 